\title{
The foundations for a global elimination programme for onchocerciasis
}

J. Anderson, H. Fuglsang, P.J.S. Hamilton, T.F.dE C. Marshall. Studies on onchocerciasis in the United Cameroon Republic I. Comparison of populations with and without Onchocerca volvulus. Transactions of the Royal Society of Tropical Medicine and Hygiene Volume 68, Issue 3, 1974, Pages 190-208. https://doi.org/10.1016/0035-9203(74)90116-3

J. Anderson, H. Fuglsang, P.J.S. Hamilton, T.F.dE C. Marshall. Studies on onchocerciasis in the United Cameroon Republic II. Comparison of onchocerciasis in rain-forest and Sudansavanna. Transactions of the Royal Society of Tropical Medicine and Hygiene Volume 68, Issue 3, 1974, Pages 209-222. https://doi.org/10.1016/0035-9203(74)90117-5

These two papers marked the start of a series of descriptions of the epidemiology of onchocerciasis in west Africa. The work was led by John Anderson, an epidemiologist, and Harald Fuglsang, an ophthalmologist, who were based at the UK Medical Research Council's Helminthiasis Research Unit, in Kumba, Cameroun in the 1970s. These papers provided the first truly systematic description of onchocerciasis, which was then and remains today a neglected tropical disease (NTD) affecting the poorest of the poor, and those 'beyond the end of the road'. It is therefore a priority disease in the RSTMH's strategy for 2017-2022 as well as being a priority for WHO in meeting the Sustainable Development Goals target of a $90 \%$ reduction in the burden of NTDs by 2030 . Both of these papers were highly influential in our understanding of the disease; the first paper determined the strength of association between various skin and eye lesions with onchocerciasis, while the second paper documented the differences in clinical manifestations between rain forest and savannah villages.

Using standardized and detailed techniques Anderson and Fuglsang found that skin and eye disease were all significantly more prevalent in infected with onchocerciasis. The prevalence of consequent blindness reached over $4 \%$ in the rain forest and $14 \%$ in the savannah villages for those aged 40 years and over. In the second paper the authors found that while there were more nodules found in infected persons in the rain forest, eye lesions were generally more common and severe in the savannah - indeed in hyperendemic savannah villages the prevalance of blindness exceeded $30 \%$ for males over 40 . The prevalence of skin lesions was about equal in the forest and the savannah villages but with different patterns of manifestation.

These studies built on the work of previous investigators including Brian Duke, Roger Crosskey, Bill Kershaw, Rodolpho Robles and Rene Le Berre. Together, these studies provided much of the epidemiological background for the creation of the Onchocerciasis Control Programme (OCP), which was launched in 1974 by WHO in collaboration with the World Bank, the United Nations Development Programme (UNDP) and the Food and Agriculture Organization (FAO) [1]. 
As the eye lesions and blindness were felt to be the most important clinical features of onchocerciasis the Programme was centred in the sahelian region of west Africa, where Anderson and Fuglsang had shown that the most severe forms of eye disease and blindness would be found. In the early years OCP operations were exclusively a vector control programme using aerial spraying. An appreciation that the blackfly vectors could fly large distances meant that the control area needed to be vast in order to prevent transmission of infection. It was well recognised from the outset that because adult Onchocerca volvulus worms were known to live for more than 10 years, the programme would have to be sustained for at least 12-15 years, Eventually the OCP covered over $1200000 \mathrm{Km}^{2}$ to protect 30 million people in 11 countries and ran for 27 years.

In 1987 Merck \& Co., Inc made what was then an unprecedented donation to the OCP of as much ivermectin for as long as it was needed and this drug treatment was subsequently combined with the larviciding control measures, and eventually largely replaced them. By the time OCP closed in 2002 transmission of the disease had been virtually eliminated in the control areas. It had been one of the greatest public health achievements of the $20^{\text {th }}$ century. OCP prevented an estimated 600000 cases of blindness, 18 million children have been born in now-controlled areas and so spared from the risk of river blindness and 25 million hectares of land have been made safe for cultivation and resettlement.

Because ivermectin can be given as a single dose once or twice a year to entire populations, OCP had pioneered the use of Community Directed Treatment (CDT) with ivermectin and this formed the basis of the successor programme - the African Programme for Onchocerciasis Control (APOC) which ran from 1995 to 2015[2]. This CDT approach has subsequently been used by a number of disease control programmes and is now a wellrecognised public health approach. By the time APOC was launched there was more recognition of the importance of skin disease and its attendant psychosocial consequences. The work of Anderson and Fuglsang had shown that since these skin lesions were to be found as much in the forest as in savannah areas the programme needed to be expanded to cover the whole area where onchocerciasis is endemic. By the time APOC closed over 118 million people had received ivermectin and over 25 million people were safe from the effects of river blindness [3]. The Onchocerciasis Elimination programme in the Americas had shown that elimination of infection was possible, and indeed in 13 of 15 foci in the New World transmission has now been interrupted. This has led the way to considering elimination of onchocerciasis in Africa, with several countries having achieved subnational elimination. APOC was succeeded in 2016 by the Expanded Special Project for the Elimination of NTDs (ESPEN) which is working to eliminate or control several NTDs as well as onchocerciasis [4].

The insights from these two papers are still central to our thinking about onchocerciasis and its control even today, 40 years later, when community treatment with ivermectin has eliminated transmission from almost all of Latin America and some parts of Africa $[5,6]$. Several challenges to achieve elimination remain including the control and elimination of onchocerciasis in Loa co-endemic areas, accelerating the interruption of transmission through development of a safe and effective macrofilaricial drug, and the public health 
challenges of scaling up prevention programmes in hypoendemic areas with low risks of eye or skin disease but where transmission still continues.

\section{References}

1. Boatin B. The Onchocerciasis Control Programme in West Africa (OCP). Ann Trop Med Parasitol. 2008;102: Suppl 1:13-7. doi: 10.1179/136485908X337427.

2. Seketeli A et al. The achievements and challenges of the African Programme for Onchocerciasis Control (APOC). Ann Trop Med Parasitol 2002: 96: Suppl 1: S15-S28. doi: 10.1179/000349802125000628.

3. World Health Organization. Weekly Epidemiological Record 2016; 91 (43): 505-514.

4. World Health Organization. Weekly Epidemiological Record 2017; 92 (45): 681-694.

5. Tekle AH et al. Impact of long-term treatment of onchocerciasis with ivermectin in Kaduna State, Nigeria: first evidence of the potential for elimination in the operational area of the African Programme for Onchocerciasis Control. Parasites \& Vectors 2012 5:28. doi: 10.1186/1756-3305-5-28.

6. Tekle $\mathrm{AH}$ et al. Progress towards onchocerciasis elimination in the participating countries of the African Programme for Onchocerciasis Control: epidemiological evaluation results. Infect Dis Poverty. 2016 Jun 27;5(1):66. doi: 10.1186/s40249-0160160-7. 